\title{
Interacting Living Polymers Confined between Two Surfaces
}

\author{
Nicolaas A. M. Besseling and Alexander V. Korobko* \\ Department of Chemical Engineering, Delft University of Technology, Julianalaan 136, 2628 BL Delft, The Netherlands
} (Received 17 July 2013; revised manuscript received 1 October 2013; published 31 October 2013)

\begin{abstract}
We present predictions on the equilibrium behavior of solutions of living polymers confined in a gap between surfaces, including the ensuing potential of mean force between those surfaces (the disjoining potential). We highlight the occurrence of a transition upon narrowing the gap, which arises from a cooperative simultaneous increase of the local density and degree of polymerization. At this transition, many properties of the confined solution, including the disjoining potential, change by orders of magnitude over a minute change of the surface separation. These results were obtained owing to two extensions to a previously introduced self-consistent field-propagator formalism. (i) We derive this formalism from a free-energy functional of the distribution of chain lengths and configurations. This enables evaluation of thermodynamic properties, including the disjoining potential. (ii) We solved for a system confined between two surfaces.
\end{abstract}

DOI: 10.1103/PhysRevLett.111.186103

PACS numbers: 68.08.- p, 68.35.Rh, 81.16.Fg, 82.70.Dd

Chains of monomers connected by reversible interactions of $\sim 10 \mathrm{kT}$ are known as living polymers [1,2]. In living polymers, bonds are formed and broken continuously, and the chain-length distribution adapts to conditions. The equilibrium chain-length distribution in a homogeneous solution follows a simple exponential law with the mean chain length governed by the binding constant and the monomer concentration [3]. Some classical examples of living polymers are actin [4], and liquid sulfur [5]. Also wormlike micelles $[6,7]$ usually behave as living polymers. The boom of supramolecular chemistry of the last decade produced many novel living polymers (often called supramolecular polymers or dynamic polymers) $[1,2,8,9]$ and incited a renewed interest in the collective physical properties of this interesting class of soft matter [10-12].

As with "ordinary" polymers [13], in which bonds between the units are covalent and of an effectively permanent nature, the presence of a small amount of living polymer in a complex (e.g., colloidal) fluid may have a pronounced influence on the behavior of the fluid as a whole [14]. Hence, living polymers can be applied to tune such behavior. This relevance for complex soft-matter systems motivated the present study on living-polymer solutions confined between two surfaces. Confining surfaces have a profound influence upon the local structure of the solution [15], and conversely, the presence of the living polymers contributes to the interaction potential of mean force between the surfaces (often called "disjoining potential") [16]. Such interactions between surfaces are crucial for the behavior of colloidal systems, as the interactions between colloidal particles are essentially the interactions between their surfaces [17]. For distances that are considerably smaller than the radii of curvature of the surfaces of, e.g., colloidal particles, the Derjaguin approximation $[17,18]$ provides a simple relation between the normalized disjoining potential $\Omega^{d} / A$ between two planar surfaces of area $A$ and the normalized disjoining force $F^{d} / R$ between curved surfaces with characteristic radius $R$ : e.g., $F^{d} / R=\pi \Omega^{d} / A$ for the disjoining force between two (colloidal) spheres with radius $R$, or $F^{d} / R=2 \pi \Omega^{d} / A$ for the disjoining force between crossed cylinders with radius $R$ or between a planar surface and a sphere with radius $R$. The latter geometries are used in experimental setups for direct measurements of disjoining forces, in a surface-force balance or a colloid-probe atomic force microscopy setup, respectively, [19,20].

In the present Letter, several novel elements come together. We formulate a free-energy functional of the distribution of chain lengths and configurations, and we establish that the self-consistent field-propagator formalism for living polymers, which was introduced before as the starting point of theoretical analysis [21], derives from this functional. Substitution of the equilibrium distribution into the functional yields all relevant thermodynamic properties of the interfacial system. Most importantly, this enables the calculation of the disjoining potential (the potential of mean force between surfaces) due to the presence of living polymers. Whereas in a previous paper the self-consistent segment-density distribution was obtained for a solution adjoining a single surface [21], in this Letter we provide numerical results for living-polymer solutions confined in a gap between two parallel surfaces, supplemented with approximate analytical expressions. This enables us to analyze the consequences of the interference of the surface layers of the two surfaces. Our analysis reveals at low adsorption strengths the occurrence of a second-order transition at which properties of the confined fluid, including the ensuing interaction between the surfaces, change drastically upon a minute change of the separation between the surfaces.

In this Letter we will provide a concise outline of the theory. A complete derivation will be published shortly. 
We examine a solution of living polymers confined in a gap between two parallel walls with area $A$, (which will be taken to be infinite, so that edge effects can be ignored). This confined solution is considered to be in equilibrium with a homogeneous reservoir solution, with a fixed monomer chemical potential $\mu$. This is representative of the situation in a colloidal system or with a typical surfaceforce measurement. The surfaces have a short-range interaction with the polymer segments, quantified by $u^{\sigma}(z)$, where $z$ is the coordinate normal to the surfaces. In this Letter the two surfaces are the same. It is straightforward however to generalize for asymmetric situations. The gap extends from a surface at $z=0$ to the surface at $z=D$. The solution in the gap is assumed to be homogeneous in the lateral direction. As a generalization of the single-particle density profile for simple particles, $\rho_{N}\left(\mathbf{z}^{(N)}\right)$ denotes the number density of chains with degree of polymerization $N$ and "configuration" $\mathbf{z}^{(N)}$ (specifying the $z$-positions of all $N$ segments of the chain). Obviously, from a complete distribution $\left\{\rho_{N}\left(\mathbf{z}^{(N)}\right)\right\}$ the corresponding overall segmentdensity profile $\rho(z)$ follows directly. Expressions for the equilibrium distribution $\left\{\boldsymbol{\rho}_{N}\left(\mathbf{z}^{(N)}\right)\right\}$ are obtained by minimization of the grand potential functional

$$
\begin{aligned}
& \frac{\Omega\left(\left\{\rho_{N}\left(\mathbf{z}^{(N)}\right)\right\}, A, D, T\right)}{A k T} \\
& =\int_{0}^{\infty} \int_{0}^{D} \rho_{N}\left(\mathbf{z}^{(N)}\right)\left(\ln \frac{\rho_{N}\left(\mathbf{z}^{(N)}\right) v_{0}}{\omega_{N}\left(\mathbf{z}^{(N)}\right)}-1+\frac{E}{k T}\right) d \mathbf{z}^{(N)} d N \\
& \quad+\frac{v}{2} \int_{0}^{D}(\rho(z))^{2} d z+\frac{1}{k T} \int_{0}^{D} u^{\sigma}(z) \rho(z) d z \\
& \quad-\frac{\mu}{k T} \int_{0}^{D} \rho(z) d z
\end{aligned}
$$

where $k$ is Boltzmann's constant and $T$ the temperature. The first contribution represents the free energy of a mixture of ideal chains of different length and configuration, where $\omega_{N}\left(\mathbf{z}^{(N)}\right)$ reflects the number of possible realizations of configuration $\mathbf{z}^{(N)}$. E is the so-called scission energy, and $v_{0}$ denotes the "binding volume". The monomermonomer binding constant is controlled by the product $v_{0} \exp (E / k T)$. The second contribution accounts for the excluded-volume interactions between segments, where $v$ is the so-called excluded-volume parameter (second virial coefficient between segments), and the third term appears due to the surface field $u^{\sigma}$ interacting with segments. $u^{\sigma}$ generally diverges at $z=0$ and at $z=D$ as segments cannot penetrate the surfaces. In addition there may be attractive wells adjoining the repulsive region.

We established that the Euler-Lagrange equation $\partial \Omega / \partial \rho_{N}\left(\mathbf{z}^{(N)}\right)=0$, for the equilibrium distribution of chain lengths and configurations, can be recast in the form of a previously proposed propagator formalism for the statistical weight of ends of chains of increasing length $N[21,22]$ (where "chain coordinate" $N$ comes in the place where in diffusion or quantum problems time appears) [23,24]. As derived before [21,22], owing to the exponential chain-length distribution in the reservoir solution, the segment-density profile for living polymers can be expressed as $\rho(z)=\rho_{0} g(z)^{2}$, where $\rho_{0}$ is the segment density in the reservoir and $g(z)$ is the Laplace-transformed propagator, which is given by the Laplace-transformed Edwards equation

$$
R_{0}^{2} \frac{d^{2} g(z)}{d z^{2}}=x g(z)^{3}+(1-x) g(z)-1 .
$$

Here, $R_{0}=b \sqrt{\langle N\rangle_{0} / 6}$, where $b$ is the mean-square segment length, is the radius of gyration of an unperturbed coil with degree of polymerization $\langle N\rangle_{0}$, being the mean degree of polymerization in the reservoir solution. The excludedvolume parameter is hidden in $x=v \rho_{0}\langle N\rangle_{0}$, which value distinguishes the dilute regime $x \ll 1$ from the excludedvolume dominated marginal solution $x \gg 1$. Physically, $g(z)$ has the meaning of a statistical weight of chain ends to be located at $z$, integrated over all chain lengths, and the density distribution of chain ends is simply $2 \rho_{0} g(z) /\langle N\rangle_{0}$. As the average degree of polymerization is just the ratio between the total number of segments over the total number of chains (which is half the number of ends), we see that the average degree of polymerization in the gap is given by $\langle N\rangle=\langle N\rangle_{0} \int_{0}^{D} g(z)^{2} d z / \int_{0}^{D} g(z) d z$.

Following de Gennes [24], the effects of the segmentsurface interactions can be accounted for by boundary conditions at the walls: $d g(z) / d z=-c g(z)$ at $z=0$; and $d g / d z=c g$ at $z=D$, where $c$ is a measure of the adsorption strength. The larger $c$ value, the stronger the adsorption; $c^{-1}$ is the the so-called adsorption or extrapolation length. As shown in previous publications on layers adjoining a single surface, negative adsorption (depletion) occurs if $c<0$, weak positive adsorption occurs if $0<$ $c R_{0}<1$, and strong adsorption if $c R_{0}>1$ [21]. In this Letter, we focus on the weak-adsorption regime as this gives rise to an interesting second-order transition upon changing the distance between the two confining surfaces.

Substitution of the expression for the equilibrium distribution $\left\{\rho_{N}\left(\mathbf{z}^{(N)}\right)\right\}$ back into Eq. (1) yields an expression for the equilibrium grand potential: $\Omega / A k T=$ $-\langle N\rangle_{0}^{-1} \int_{0}^{D} \rho(z) d z-\frac{1}{2} v \int_{0}^{D} \rho(z)^{2} d z$ and subsequently of the interfacial excess grand potential $\Omega^{\sigma} \equiv \Omega-\Omega_{0}$, where $\Omega_{0}$ is the grand potential of a homogeneous solution with reservoir concentration $\rho_{0}$ and volume $D A$. The disjoining potential is $\Omega^{d} \equiv \Omega^{\sigma}(D)-\Omega^{\sigma}(\infty)$.

Figure 1 presents normalized segment-density profiles in the gap for different distances between surfaces. For large $D / R_{0}$ ratios, the profiles converge to those at a single surface, which were presented and discussed in an earlier paper [21]. For small distances between surfaces when $D / R_{0}<1$, the segment-density profiles are almost uniform, with relatively small concentration gradients across the gap. 


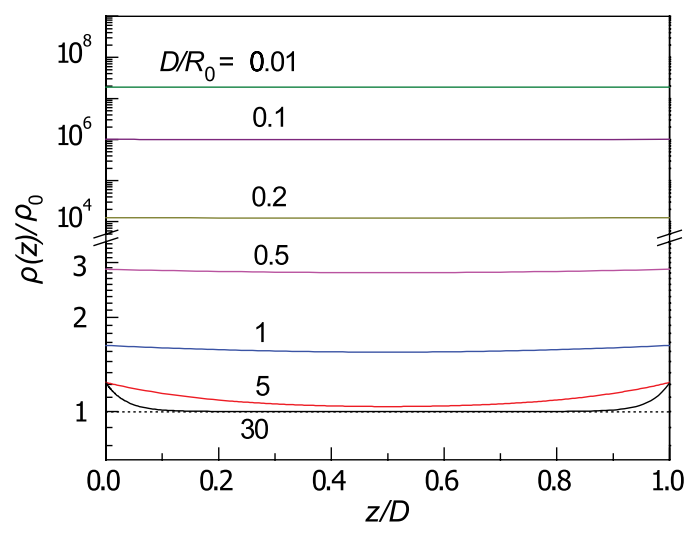

FIG. 1 (color online). Segment-density profiles between two adsorbing surfaces separated by various distances $D / R_{0}$, for $c R_{0}=0.1$. All profiles are for $x=10^{-6}$. The transition occurs at $D / R_{0} \simeq 0.2$, where the density rapidly increases with decreasing distance between surfaces. Note the use of linear as well as logarithmic vertical axes.

The uniform character of the profiles in the regime of $D / R_{0}<1$ enables an insightful simple analytical solution to Eq. (2), which we will call the flat-profile approximation. After integration of the left- and right-hand sides of Eq. (2) over the volume, the Gauss theorem can be used to replace the volume integral of the second derivative on the lhs by a surface integral of the first derivative, for which we can substitute $-c g$ according to the surface boundary condition. Invoking now the uniform profile, $g(z)=g$, we arrive at the incomplete cubic equation

$$
x g^{3}+\psi g-1=0,
$$

with $\psi=1-x-2 R_{0}^{2} c / D$. Equation (3) minimizes the potential $x g^{4} / 4+\psi g^{2} / 2-g$ for the "order parameter" $g$. The first two terms are analogous to the Landau potential of a system exhibiting a second-order phase transition at a "reduced temperature" $\psi=\psi_{c} \equiv 0$ [25]. For the present living-polymer system, the symmetry of this Landau potential is broken by the "external-field" term -1 conjugate to $g$. In the absence of the surface effect, that is, for $c / D=0$, the Landau potential would predict a secondorder phase transition at $x=1$. However, confinement introduces the term $2 R_{0}^{2} c / D$ in the expression for $\psi$. Therefore, adsorbing surfaces shift the phase transition to a lower value of $x$.

The physically relevant (positive and real) root of Eq. (3) is $g(\psi)=\eta /(6 x)-2 \psi / \eta$, where $\eta=$ $x^{2 / 3}\left(108+12 \sqrt{3} \sqrt{\left(4 \psi^{3}+27 x\right) / x}\right)^{1 / 3}[26]$. For $\psi=0$, the solution yields the monomer density at the second-order phase transition in the Landau potential: $\rho_{c}=\rho_{0} x^{-2 / 3}$.

For $\psi \ll 0$, the term -1 in Eq. (3) is negligible, so $g^{2}=-\psi / x$ and $\rho=-\rho_{0} \psi / x$. Furthermore, $\psi \ll 0$ implies $2 R_{0}^{2} c / D \gg 1-x$; hence, $\rho=\rho_{0} R_{0}^{2} c D^{-1} x^{-1}$. Consequently, we obtain for this case of small gap width for the excess amount of segments per unit area inside the gap $\theta^{\text {ex }} \equiv \int_{0}^{D}\left(\rho(z)-\rho_{0}\right) d z \quad$ a limiting expression $\theta_{I I}^{\mathrm{ex}} / \rho_{0} \simeq 2 R_{0}^{2} c / x$. For the average degree of polymerization, we obtain $\langle N\rangle /\langle N\rangle_{0} \simeq R_{0} \sqrt{2 c / D x}$, and for the interfacial excess grand potential we obtain $\Omega^{\sigma}\langle N\rangle_{0} / A k T \rho_{0} \simeq-2 R_{0}^{4} c^{2} / D x$.

In the regime where the flat-profile approximation clearly fails, at $D / R_{0}>1$, the profile is approximated well as just the sum of two profiles associated with two separate surfaces derived according to Ref. [21]. This additivity approximation provides $\Omega^{\sigma}(\infty)$ needed to calculate the disjoining potential. For large surface separations $\left(D / R_{0}>1\right)$ and weak adsorption $\left(c R_{0}<1\right)$, the excess amount of monomers is limited by $\theta_{I}^{\mathrm{ex}} / \rho_{0}=2 \times$ $2 c R_{0}^{2} /(1+2 x)$ [21]. The ratio $\theta_{I I}^{\mathrm{ex}} / \theta_{I}^{\mathrm{ex}}=(1+2 x) / 2 x$ indicates that in marginal solutions, at $x \geq 1$, the magnitude of transition becomes small.

Figure 2 reveals the transition of $\theta^{\text {ex }}$ from $\theta_{I}^{\text {ex }}$ to $\theta_{I I}^{\text {ex }}$ at fixed surface separation upon variation of the adsorption strength $c R_{0}$. As discussed above, when $D / R_{0} \ll 1$, the transition happens at $\psi=\psi_{c}=0$, coinciding with the inflection point of the dependency. The segment-density profile remains flat after transition at high $c R_{0}$, reflecting chains to occupy all the space between surfaces uniformly. For $D / R_{0}>1$, the weak-adsorption regime is extended up to higher $c R_{0}$ values at which the transition occurs. The density profile is not flat, and the transition occurs in two adsorbed layers separated from each other by a region with reservoirlike properties $\left(\rho \simeq \rho_{0}\right)$. For this situation the flatprofile approximation Eq. (3) obviously fails, and the analysis of adsorbed layers adjoining a separate surface applies [21].

Figure 3 displays the gap-width dependencies of several properties of confined solutions of weakly adsorbing living polymers $\left(c R_{0} \leq 1\right)$ : (a) the excess amount of segments in the gap, per unit area, (b) the mean degree of

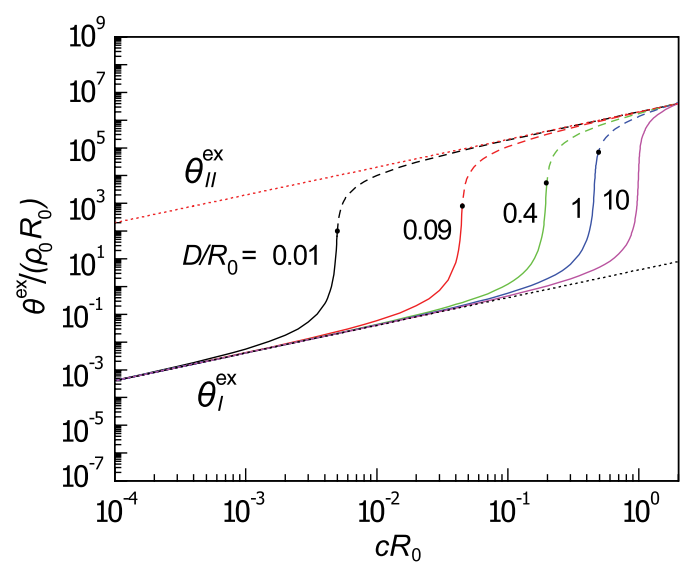

FIG. 2 (color online). The excess amount of monomers confined between adsorbing surfaces versus the adsorption parameter $c R_{0}$ at different gap widths. Dotted lines show the scaling laws discussed in the text. Solid and dashed lines correspond to $\psi>0$ and $\psi<0$, respectively. Solid circles denote $\psi=0$. 


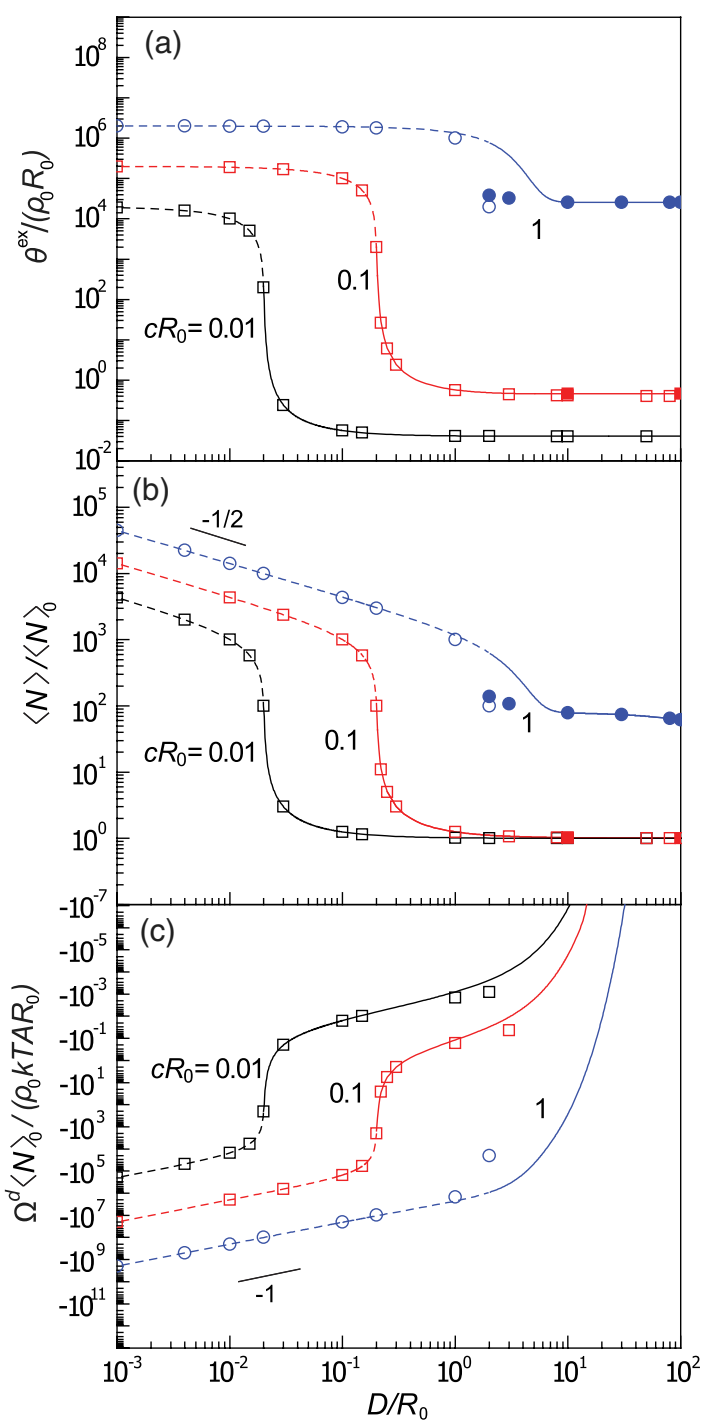

FIG. 3 (color online). Characteristics of the confined solution as a function of the normalized gap width $D / R_{0}$ for $x=10^{-6}$ and for different values of the normalized adsorption strength $c R_{0}$ : (a) normalized excess of segments, (b) average degree of polymerization, and (c) the disjoining potential. The drawn lines are exact numerical results. Open and closed symbols belong to the flat-profile and the additivity approximation, respectively. Solid and dashed lines are as in Fig. 2.

polymerization inside the gap, and (c) the disjoining potential $\Omega^{d}$ (= potential of mean force between the surfaces). The results were obtained by solving Eq. (2) numerically and from the flat-profile (open symbols) and additivity (solid symbols) approximations. Figure 3 demonstrates that both approximations work well for the regimes where they are expected to.

For large distances between the surfaces, the excess amount of segments is just twice that of an adsorption layer at a single surface as discussed in Ref. [21]. The mean degree of polymerization inside the gap approaches that of the reservoir solution when the gap width increases, because reservoirlike properties prevail in most of the gap (in the space between the adsorption layers). The disjoining potential vanishes by definition for $D / R_{0} \rightarrow \infty$.

The transition is clearly recognized in Fig. 3 at the inflection point, and the local concentration of monomers and the degree of polymerization changes tremendously with a small variation of $D / R_{0}$. It is the cooperativity between this density change and the change of the degree of polymerization that constitutes the physical mechanism of the near-second-order transition. The level to which the local density inside the gap increases is limited by the repulsive excluded-volume interactions, quantified by the parameter $v$. The transition in the confined livingpolymer solution can be regarded as a "rudimentary" capillary condensation. The latter is the phase transition upon which the contents of a capillary (e.g., a gap between two surfaces) turns from vapor to liquid. In the case of twodimensional (formation and growth of linear livingpolymer chains) rather than three-dimensional association (e.g., condensation of liquid from a vapor), a true phase transition cannot occur, but a near-second-order transition does take place. At small distances, the uniform density profile of monomers and the negative value of $\psi$ yield a constant value of $\theta^{\mathrm{ex}} / \rho_{0} R_{0}$ and scaling of $\langle N\rangle /\langle N\rangle_{0}$ and $\Omega^{d}\langle N\rangle_{0} /\left(A k T \rho_{0} R_{0}\right)$ as predicted above by means of the flat-profile approximation. Remarkably, the disjoining potential experiences a jump of several orders of magnitude at the surface separation $D \simeq 2 R_{0}^{2} c$.

In this Letter, we predict a near-second-order phase transition for living polymers confined by two surfaces. Properties of the confined system such as the density profile, the degree of polymerization, and the disjoining potential between the confining surfaces are sensitive to the degree of polymerization in the reservoir solution, the adsorption strength, and the gap width. The confined system undergoes a transition when $\rho_{0} v\langle N\rangle_{0}<1$ and $c R_{0}<1$ at the gap width corresponding to $\psi=0$. An important consequence is that the disjoining potential between the surfaces is boosted at the critical surface separation for systems for which at larger separation adsorption is quite insignificant. Our approach accounts for adaptable polydispersity and configurational freedom of chains, for the chain affinity to the surface, and for excluded-volume interactions between chains. In solutions with relatively large chains $R_{0}>D$, the transition occurs in the flat-profile regime for which the density profile inside the gap is uniform. In the opposite limit of $D>R_{0}$, a transition occurs in the separate adsorbed layers. Our results can be used to design conditions at which living polymers exert a distinct effect upon surface and intercolloidal interactions.

This work was supported by ECHO Grant No. 700.59.025 from the Netherlands Organization for Scientific Research (NWO). 
*A.Korobko@tudelft.nl

[1] L. Brunsveld, B. J. B. Folmer, E. W. Meijer, and R. P. Sijbesma, Chem. Rev. 101, 4071 (2001).

[2] M. J. Serpe and S. L. Craig, Langmuir 23, 1626 (2007).

[3] M. E. Cates and S. J. Candau, J. Phys. Condens. Matter 2, 6869 (1990).

[4] T. D. Pollard and J. A. Cooper, Annu. Rev. Biochem. 55, 987 (1986).

[5] A. V. Tobolsky and W. J. MacKnight, Polymeric Sulphur and Related Polymers (Interscience, New York, 1965).

[6] Y.-Y. Won, H. T. Davis, and F. S. Bates, Science 283, 960 (1999).

[7] M. E. Cates, J. Phys. 49, 1593 (1988).

[8] T. F. A. de Greef, G. Ercolani, G. B. W. L. Ligthart, E. W. Meijer, and R. P. Sijbesma, J. Am. Chem. Soc. 130, 13755 (2008).

[9] F. C. Mackintosh, S. A. Safran, and P. A. Pincus, Europhys. Lett. 12, 697 (1990).

[10] E.H. Feng and G. H. Fredrickson, Macromolecules 39, 2364 (2006).

[11] S. Zou, H. Schönherr, and G. J. Vancso, Angew. Chem., Int. Ed. 44, 956 (2005).

[12] M.E. Helgeson and N. J. Wagner, J. Chem. Phys. 135, 084901 (2011).
[13] G. J. Fleer, Adv. Colloid Interface Sci. 159, 99 (2010).

[14] J. van der Gucht, N. A. M. Besseling, and M. A. Cohen Stuart, J. Am. Chem. Soc. 124, 6202 (2002).

[15] C. E. Woodward and J. Forsman, Phys. Chem. Chem. Phys. 13, 5764 (2011).

[16] C.E. Woodward and J. Forsman, Macromolecules 42, 7563 (2009).

[17] J. N. Israelachvilli, Intermolecular and Surface Forces (Academic Press, New York, 2011), 3rd ed.

[18] B. V. Derjaguin, Kolloidn. Zh. 69, 155 (1934).

[19] W. A. Ducker, T. J. Senden, and R. M. Pashley, Nature (London) 353, 239 (1991).

[20] W. Knoben, N. A. M. Besseling, and M. A. Cohen Stuart, Phys. Rev. Lett. 97, 068301 (2006).

[21] J. van der Gucht, N. A. M. Besseling, and G. J. Fleer, Macromolecules 37, 3026 (2004).

[22] J. van der Gucht, N.A.M. Besseling, and G. J. Fleer, J. Chem. Phys. 119, 8175 (2003).

[23] S. F. Edwards, Proc. Phys. Soc. 85, 613 (1965).

[24] P. G. de Gennes, Rep. Prog. Phys. 32, 187 (1969).

[25] L.D. Landau and E. M. Lifshitz, Statistical Physics (Butterworth-Heinemann, Oxford, 1980), 3rd ed.

[26] G. A. Korn and T. M. Korn, Mathematical Handbook for Scientists and Engineers (McGraw-Hill Book Company, New York, 1968), 2nd ed. 